\title{
Pulse wave velocity with 4D flow MRI: Systematic differences and age-related regional vascular stiffness
}

Petter Dyverfeldt, Tino Ebbers and Toste Länne

\author{
Linköping University Post Print
}

Tweet

N.B.: When citing this work, cite the original article.

Original Publication:

Petter Dyverfeldt, Tino Ebbers and Toste Länne, Pulse wave velocity with 4D flow MRI: Systematic differences and age-related regional vascular stiffness, 2014, Magnetic Resonance Imaging, (32), 10, 1266-1271.

http://dx.doi.org/10.1016/j.mri.2014.08.021

Copyright: Elsevier

http://www.elsevier.com/

Postprint available at: Linköping University Electronic Press

http://urn.kb.se/resolve?urn=urn:nbn:se:liu:diva-112805 
Pulse Wave Velocity with 4D Flow MRI: Systematic Differences and AgeRelated Regional Vascular Stiffness

Petter Dyverfeldt $(\mathrm{PhD})^{1,2}$, Tino Ebbers $(\mathrm{PhD})^{1-3}$, Toste Länne(MD, $\left.\mathrm{PhD}\right)^{1,2,4}$

1. Division of Cardiovascular Medicine, Department of Medical and Health Sciences, Linköping University, Linköping, Sweden.

2. Center for Medical Image Science and Visualization (CMIV), Linköping University, Linköping, Sweden.

3. Division of Media and Information Technology, Department of Science and Technology / Swedish e-Science Research Centre (SeRC), Linköping University, Linköping, Sweden.

4. Department of Cardiovascular Surgery, Linköping University Hospital, County Council of Östergötland, Linköping, Sweden.

Correspondance:

Petter Dyverfeldt

KVM, Huvudblocket, Plan 12

Campus US

Linköping University

SE-581 83 Linköping, Sweden.

E-mail: petter.dyverfeldt@ liu.se

Phone: +46 101033353 


\section{ABSTRACT}

Purpose: The objective of this study was to compare multiple methods for estimation of PWV from 4D flow MRI velocity data and to investigate if 4D flow MRI-based PWV estimation with piecewise linear regression modeling of travel-distance vs. travel time is sufficient to discern age-related regional differences in PWV.

Methods: 4D flow MRI velocity data were acquired in 8 young and 8 older (age: $23 \pm 2$ vs. $58 \pm 2$ y.o.) normal volunteers. Travel-time and travel-distance was measured throughout the aorta and piecewise linear regression was used to measure global PWV in the descending aorta and regional PWV in three equally sized segments between the top of the aortic arch and the renal arteries. Six different methods for extracting travel-time were compared.

Results: Methods for estimation of travel-time that use information about the whole flow waveform systematically overestimate PWV when compared to methods restricted to the upslope-portion of the waveforms $(\mathrm{p}<0.05)$. In terms of regional PWV, a significant interaction was found between age and location $(\mathrm{p}<0.05)$. The age-related differences in regional PWV were greater in the proximal compared to distal descending aorta.

Conclusion: Care must be taken as different classes of methods for the estimation of traveltime produce different results. 4D flow MRI-based PWV estimation with piecewise linear regression modeling of travel-distance vs. travel time can discern age-related differences in regional PWV well in line with previously reported data.

Key words: Pulse wave velocity, MR flow imaging, vascular stiffness, 4D flow MRI, phasecontrast MRI, ageing. 


\section{INTRODUCTION}

Aortic pulse wave velocity (PWV) is a marker of vascular stiffness and a strong and independent predictor of cardiovascular morbidity and mortality ${ }^{1-4}$.

Catheter-based pressure measurements are considered gold standard for PWV estimation but this method is rarely used due to its invasiveness. Clinically, a combination of tonometry or ultrasound and measuring-tape is used to assess carotid-to-femoral PWV. The invasiveness of the gold standard technique and the limitations of the current clinical approach have prompted the development of alternative means of estimating PWV ${ }^{5}$. Notably, MRI has evolved as a promising method ${ }^{6-18}$ The merits of MRI for the estimation of PWV include the capabilities of obtaining: 1) angiographic images that permit accurate determination of vascular distance and 2) an arbitrary number of velocity or flow waveforms at any location in the body. MRbased PWV estimation has primarily been done with $2 \mathrm{D}$ cine phase-contrast (PC) MRI ${ }^{6,9-}$ 11,13. Another method that has been explored is 1D Fourier velocity encoding (FVE) MRI, which permits high temporal resolution but is limited to applications in straight vessel segments ${ }^{7,8,15,16}$.

Most of the existing data on in-vivo PWV concerns global aortic PWV. However, vascular stiffness and thus PWV is known to vary regionally along the vasculature ${ }^{19}$. This may be particularly interesting with respect to focal diseases such as thoracic or abdominal aortic aneurysms. A few previous studies have addressed regional PWV invasively using catheterbased pressure measurements and non-invasively using 2D cine PC-MRI or 1D Fourier velocity encoding (FVE) MRI ${ }^{10,16,20}$. 
The traditional approach to PWV estimation is based on the distance ("travel-distance") between two locations in the vasculature and the temporal shift between velocity, flow or pressure waveforms recorded at these locations ("travel-time"). This "time to travel a fixed distance"-approach, essentially treats travel-distance as a discrete variable and therefore high (temporal) resolution is crucial for the single continuous variable travel-time. Shorter vascular distance, as used in regional PWV assessment, necessitates an even higher temporal resolution. There exist multiple methods for the estimation of travel-time and it is known that the choice of method can impact the results ${ }^{18,20-22}$. In principle, PWV can also be estimated in the opposite way, i.e. by locating the pulse wave at the two instances in time. In such a "distance travelled in a fixed time" approach, the spatial resolution would be crucial, as well as an appropriate algorithm for spatial pulse wave detection. The "distance travelled in a fixed time" approach could be advantageous for assessment of spatial variations of PWV.

Recently, some studies have explored PWV estimation based on 3D cine PC-MRI (“4D flow MRI") ${ }^{14,17,18}$. The most interesting feature of 4D flow MRI for PWV estimation is its full 4D spatiotemporal coverage of velocity data. With this 4D coverage, both travel-time and traveldistance can be treated as continuous variables. Consequently, 4D flow MRI meets the prerequisites for PWV assessment using both the "time to travel a fixed distance" and the "distance travelled in a fixed time" approach. Optimally, these approaches are combined in one estimate of the PWV. One way to exploit the 4D coverage is based on the extraction of flow waveforms at multiple locations throughout a vessel of interest. In this approach, the travel-distance variable is mapped based on 3D vascular anatomy derived from the 4D flow MRI data. The travel-time variable is calculated by conventional transit-time methods. The relationship between the variables can be modeled with piecewise linear regression, for example. 
The objective of this study was to compare multiple methods for estimation of PWV from 4D flow MRI velocity data and to investigate if 4D flow MRI-based PWV estimation with piecewise linear regression modeling of travel-distance vs. travel time is sufficient to discern age-related regional differences in PWV.

\section{MATERIALS AND METHODS}

\subsection{Study population}

8 young (age: $23 \pm 2$ years, range 21-26 years) and 8 older (age: $58 \pm 2$ years, range 55-60 years) normal male volunteers were included in the study (see Table 1). All volunteers were normotensive and non-smokers, had no history of cardiovascular disease, and did not take any medication. The volunteers were also asked to refrain from intake of any vasoactive substances (including caffeine) at the day of the MRI study. The local ethics committee approved the study and written informed consent was obtained from all participants.

\subsection{D Flow MRI}

$3 \mathrm{D}$ cine phase-contrast (4D flow) MRI velocity data of the aorta were acquired using a $1.5 \mathrm{~T}$ scanner (Philips Achieva, Philips Healthcare, Best, the Netherlands) with a retrospectively electrocardiogram-gated gradient-echo sequence with interleaved three-directional velocity encoding ${ }^{23}$. Imaging parameters included $\mathrm{TE}=2.8-3.1 \mathrm{~ms}, \mathrm{TR}=4.9-5.4 \mathrm{~ms}$, flip angle $=6$ degrees, velocity encoding range $(\mathrm{VENC})=160-200 \mathrm{~cm} / \mathrm{s}$, parallel imaging reduction factor $(\mathrm{SENSE})=2, \mathrm{k}$-space segmentation factor $=2$, matrix size $=128-144 \times 128-144$, 3D field-ofview: $300-420 \times 300-420 \times 70 \mathrm{~mm}^{3}$, voxel size $=2.3-2.8 \mathrm{~mm}$ isotropic. Respiratory motion artifacts were suppressed by navigator gating with an acceptance window of $7 \mathrm{~mm}$. Nominal scan time was 896-1000 heartbeats and the respiratory navigator efficiency was around 60\%. 
The acquired temporal resolution was $39-43 \mathrm{~ms}$ and the retrospectively gated data were reconstructed into 32 time frames on the scanner. Maxwell effects were corrected on the scanner and offline processing was applied to correct for phase-wraps and background phaseerrors ${ }^{24,25}$.

\subsection{Semi-automatic PWV estimation}

A semi-automatic approach to PWV estimation was implemented in Matlab (Mathworks Inc., Natick, MA, United States) (Figure 1). The approach used here is similar to that used in previous 4D flow MRI studies in that is exploits the volumetric nature of the data and extracts complete pulsatile flow waveforms at multiple locations ${ }^{14,18}$. Similar methods have also been used with 2D in-plane PC-MRI and 1D FVE, where velocity-time profiles rather than complete pulsatile flow waveforms are measured ${ }^{8,26}$. First, a 3D segmentation of the aorta was obtained by manual delineation in angiographic images which were generated by taking the systolic time-average of the product between velocity magnitude (i.e. speed) and MR signal intensity to the power of 1.2. Next, the centerline of the aorta was extracted by using a fast-marching algorithm ${ }^{27}$. Based on the centerline, flow vs. time waveforms in planes perpendicular to the aorta were extracted automatically with $1 \mathrm{~mm}$ spacing throughout the aorta. $1 \mathrm{~mm}$ spacing corresponds to a finer resolution than the native spatial resolution of the source images and represents a way to avoid loss of information due to spatial undersampling of the travel-distance variable. The flow waveforms were upsampled by a factor of 40 using spline interpolation. The travel-time and travel-distance variables were calculated using transit-time algorithms and distances along the centerline, respectively. This resulted in a travel-time vs. travel-distance graph that could be interrogated for global and regional PWV estimation. Global PWV (PWV global $)$ in the descending aorta was obtained by linear fitting of travel-time vs. travel-distance from the top of the arch to the renal arteries. Further, the aortic 
region between the top of the arch and the renal arteries was divided into three equally sized segments: the proximal, mid and distal suprarenal descending aorta. Regional PWV in these three segments was calculated in the same way as the global PWV.

Six different methods for the estimation of the travel-time variable were included:

- $\quad$ Time to foot (TTF) method

Tracks the point at which a line fitted to the upslope of the flow curve crosses the base of the flow waveform (i.e. foot of waveform) ${ }^{20,28,29}$. The upslope region of the flow waveform was defined as the region between $20 \%$ and $70 \%$ of the peak flow rate. The base of the flow waveform was defined as the average diastolic flow rate.

- $\quad$ Time to peak upslope (TTU) method

Tracks the point of peak first derivative at the upslope of the waveform ${ }^{30}$.

- Time to foot method \#2 (TTF2)

Tracks the point on the flow waveform that corresponds to $20 \%$ of the flow rate at the point of the maximum derivative of the upslope portion of the waveform ${ }^{21}$. The maximum derivative was obtained as for the TTU method.

- Fourier analysis (FA) method

Estimates travel-time based on the phase-shift between two waveforms ${ }^{31}$. A line is fitted to the low frequency components of the quotient of the Fourier transforms of the two waveforms. The slope of this line gives the travel-time: travel-time $=$ slope $/ 2 \pi$. In the present study, the 1-3 Hz range was considered.

- Cross correlation (XC) method

Estimates the travel-time as the time-shift that results the maximum cross correlation between two waveforms ${ }^{10,12}$. In the present study, cross correlation was applied to the complete flow waveform. 
- Center of mass (COM) method

Tracks the center-of-mass of the main lobe of the flow waveform. The main lobe was defined as the portion between $20 \%$ of the peak flow rate at the upslope and $20 \%$ of the peak flow rate at the downslope. To our knowledge this method has not been previously published.

It may be noted that the first three methods (TTF, TTU and TTF2) operate on the upslope of the flow waveform. The last three methods (FA, XC, COM), on the other hand, use information about larger parts of the waveform. As pointed out in previous studies, the latter class of methods can be expected to be more sensitive to the presence of reflected waves $20,21,29$

\subsection{Data analysis}

Results on global and regional PWV in the descending aorta are reported for all six methods for the estimation of the travel-time variable.

Statistical evaluations of the effects of age and location (age-related regional PWV), as well as detailed inter-methods comparisons, were carried out for the TTF and XC methods. These methods were chosen because they represent two of today's most frequently used methods. The TTF method is used with multiple modalities and has frequently been considered the most accurate method. The XC method was introduced more recently and has gained popularity due to its robustness inherent to the use of the complete flow waveform. Additionally, age-related global and regional PWV in the ascending aorta were compared against corresponding data available in the literature. 
Numerical results are reported as mean \pm one standard deviation, unless otherwise noted. One-way analysis of variance (ANOVA) with Tukey's honestly significant difference (HSD) post-hoc analysis was used to assess the difference between the different methods for extracting travel-time. The difference between the TTF and XC methods was further assessed by linear regression and Bland-Altman analysis ${ }^{32}$. Two-way ANOVA was used to assess the interaction between age and location. All calculations were done in Matlab (Mathworks, Natick, MA, USA).

\section{RESULTS}

The PWV for the different age groups, locations and methods evaluated in this study are shown in Table 2. One-way ANOVA revealed a significant difference in $\mathrm{PWV}_{\text {global }}$ among the six different methods for extracting PWV (one-way ANOVA, P $<0.001$. Post-hoc tests further showed that $\mathrm{PWV}_{\text {global_FA, }} \mathrm{PWV}_{\text {global_XC }}$ and $\mathrm{PWV}_{\text {global_COM }}$ were higher than

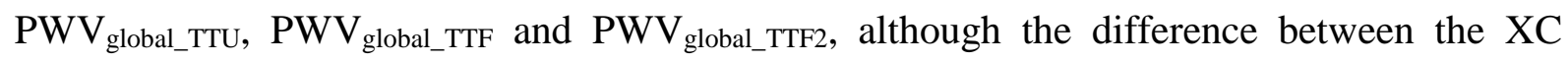
method vs. TTU and TTF2 methods was not significant. Moreover, there were no significant differences between $P W V_{\text {global_FA }}$ vs. PWV global_XC vs. PWV global_com or PWV global_TTU vs. PWV $V_{\text {global_TTF }}$ Vs. PWV $V_{\text {global_TTF2, }}$ respectively, for any of the two age groups.

A direct comparison between the XC method and TTF method with linear regression and Bland-Altman analysis revealed that PWV_global_XC tended to be higher than PWV_global_TTF. The estimated linear regression function was $\mathrm{PWV}_{\text {global_XC }}=2.37+0.97 * \mathrm{PWV}_{\text {global_TTF }}\left(\mathrm{r}^{2}=\right.$ 0.76), where both the slope and intercept were significantly different from zero. In the BlandAltman analysis, bias $\pm 2 \mathrm{SD}$ was $2.21 \pm 1.01 \mathrm{~m} / \mathrm{s}$. The same tendency can be observed for regional PWV, as seen in Table 1. 
Paired two-tailed t-tests demonstrated that $\mathrm{PWV}_{\text {global }}$ was significantly higher in the older compared to the younger volunteers with both the XC method and TTF method. Further, a significant interaction between age and site of increased PWV was found for both methods (two-way ANOVA, P < 0.05). Profile plots of PWV versus location (see Figure 2) demonstrate that the age-related differences in regional PWV were greatest in the proximal descending aorta and smallest in the distal suprarenal descending aorta. These age-related changes in PWV were significant for the proximal descending aorta and the mid descending aorta for both the XC and TTF methods (one-way ANOVA, P $<0.01$ ). The age-related difference in distal suprarenal descending aorta did not reach significant levels for any of the methods (one-way ANOVA).

Age-related regional PWV with the TTF method in the young vs. older subjects were $3.7 \pm$ 0.4 vs. $8.8 \pm 3.5,3.3 \pm 0.5$ vs. $7.0 \pm 2.1$, and $4.5 \pm 0.8$ vs. $5.2 \pm 1.5 \mathrm{~m} / \mathrm{s}$ for the proximal, mid and distal descending aortic segments, respectively. The corresponding numbers for the XC method were $4.2 \pm 0.7$ vs. $10.2 \pm 3.6,5.7 \pm 1.6$ vs. $10.6 \pm 3.8$, and $4.6 \pm 0.8$ vs. $6.9 \pm 1.7$. A comparison between these values of age-related regional PWV and those reported in previous studies is shown in Figure 3. The TTF method appears to match literature data better than the XC method.

\section{DISCUSSION}

4D flow MRI allows PWV assessment with both the traditional "time to travel a fixed distance" approach and the opposite "distance travelled in a fixed time" approach. In the present study employed a combined approach based on the extraction of flow waveforms throughout a vessel of interest. In this approach, the travel-distance variable is mapped based on 3D vascular anatomy derived from the 4D flow MRI data and the travel-time variable is 
calculated by conventional transit-time methods. As the choice of method can impact the results, six different methods for estimation of travel-time were evaluated in the present study. The findings of the inter-method comparisons suggest that the six methods can be divided into two different groups. One group that operates on the upslope portion of the waveform (TTU, TTF, and TTF2) and one group that operates on larger parts of the waveform (FA, XC, and $\mathrm{COM}$ ). As previous studies have pointed out, the latter class of methods is more sensitive to the presence of reflected waves ${ }^{20,21,29}$. All methods in the latter group produced $\mathrm{PWV}_{\text {global }}$ values that were higher than those in the former group. There were no significant differences within the groups of methods. A direct comparison between the TTF and XC methods indicated that the XC method overestimates $\mathrm{PWV}_{\text {global }}$ by about $2 \mathrm{~m} / \mathrm{s}$ compared to the TTF method. These findings indicate that care must be taken when interpreting PWV values obtained with different methods for estimation of travel-time. The age-related regional PWV obtained with the TTF method better matched the patterns of age-related regional PWV that have been reported previously ${ }^{10,16,20}$.

To our knowledge, the present study is the first to report 4D flow MRI-based estimation of age-related regional PWV. Our results on age-related regional PWV obtained with the TTF method agree well with previously reported data and show that the PWV in the descending aorta is homogeneous in young subjects and markedly heterogeneous in older subjects ${ }^{10,16,20}$. We also found that the PWV increases more rapidly with age in the proximal compared to distal suprarenal descending aorta. A structural explanation for this observation may be derived from the fact that the ratio between elastin to collagen decreases towards the distal parts of the aorta, in combination with the life-long fragmentation of elastin ${ }^{29}$. It should be noted that this and previous studies have observed a trend towards increased PWV with age also in the distal aorta, although the studies have been too small to statistically verify this 
difference ${ }^{10,16,20}$. However, this trend has been confirmed by larger-scale investigations of local stiffness in the distal aorta ${ }^{33}$.

In 4D flow MRI, high temporal resolution is costly in terms of increased scan time. Consequently, the acquired temporal resolution of 4D flow MRI is often low compared to other techniques used to estimate PWV. However, 4D flow MRI offers full 3D coverage. High temporal resolution is crucial for the conventional "time to travel a fixed distance"approach, which aims to determine travel-time based on the timing of the pulse wave at two discrete locations. Low precision in the assessment of timing (i.e. if temporal resolution is low, or if data is noisy) directly impacts the precision of the PWV estimate. The situation becomes different when the distance in not regarded as fixed, as in the present study and previous 4D flow MRI studies ${ }^{14,18}$. Treating distance as a continuous variable enables the use of analysis locations along the entire aorta. Clearly, temporal resolution and noise define the precision with which the timing of the pulse wave can be determined at each specific location. However, when the timing information from all locations is combined (here using linear regression, see Figure 1C), the significance of the lack of precision of each individual observation, and thus the sensitivity to temporal resolution, is reduced. In the present study, we found that 4D flow MRI-based regional PWV closely matches regional PWV data available in the literature (see Figure 3). An age-based comparison of our results on global PWV in the descending aorta corresponding high-temporal resolution PWV data available in the literature provides complementary support of the promising performance of $4 \mathrm{D}$ flow MRI (see Table 3). This accord well with previous comparisons between 4D flow MRI and methods with higher temporal resolution ${ }^{14,18}$. Nevertheless, further studies are needed to assess the impact of temporal resolution, as well as spatial resolution, on PWV estimates. Future work should also assess the minimum vascular distance along which PWV can be 
calculated for different spatial and temporal resolutions. Another intriguing aspect for future work is to improve the combination of the "time to travel a fixed distance" and "distance travelled in a fixed time" approaches. Linear regression modeling of the relationship between the travel-distance and travel-time variables, as used here, may not be the optimal way to exploit the full 4D spatiotemporal coverage.

An important limitation of the present study is that we did not have access to gold standard catheter-based PWV estimates in our normal volunteers. Neither did our imaging protocol include 1D FVE or multi-planar 2D cine PC-MRI. We are therefore unable to report on the precision of 4D flow MRI-based PWV estimation relative to the gold-standard method as well as to other MRI methods. Consequently, the comparison of different methods for the estimation of travel-time may only be valid for the type of 4D flow data and data processing used in this study.

\section{Conclusion}

4D flow MRI-based PWV estimation with piecewise linear regression modeling of traveldistance vs. travel time can discern age-related differences in regional PWV well in line with previously reported data. However, care must be taken as different classes of methods for the estimation of travel-time based on flow waveforms obtained by $4 \mathrm{D}$ flow MRI produce different results. Methods that use information from the complete flow waveform estimate higher PWV than methods that are restricted to the upslope portion of the flow waveform.

\section{ACKNOWLEDGEMENT}

The authors acknowledge Mats Fredriksson for support with the statistical analysis. Grant support is acknowledged from the Swedish Research Council (PD, TE), the Swedish Heart- 
Lung Foundation (TL), and the Medical Research Council of Southeast Sweden (FORSS) (PD, TL). 


\section{REFERENCES}

1. Safar ME, Henry O, Meaume S. Aortic pulse wave velocity: an independent marker of cardiovascular risk. The American Journal of Geriatric Cardiology 2002;11(5):295-304.

2. Sutton-Tyrrell K, Najjar SS, Boudreau RM, Venkitachalam L, Kupelian V, Simonsick EM, Havlik R, Lakatta EG, Spurgeon H, Kritchevsky S. Elevated aortic pulse wave velocity, a marker of arterial stiffness, predicts cardiovascular events in well-functioning older adults. Circulation 2005;111(25):3384-3390.

3. Willum-Hansen T, Staessen JA, Torp-Pedersen C, Rasmussen S, Thijs L, Ibsen H, Jeppesen J. Prognostic value of aortic pulse wave velocity as index of arterial stiffness in the general population. Circulation 2006;113(5):664-670.

4. Mancia G, De Backer G, Dominiczak A, Cifkova R, Fagard R, Germano G, Grassi G, Heagerty AM, Kjeldsen SE, Laurent S. 2007 Guidelines for the management of arterial hypertension The Task Force for the Management of Arterial Hypertension of the European Society of Hypertension (ESH) and of the European Society of Cardiology (ESC). European heart journal 2007;28(12):1462-1536.

5. Segers P, Kips J, Trachet B, Swillens A, Vermeersch S, Mahieu D, Rietzschel E, De Buyzere M, Van Bortel L. Limitations and pitfalls of non-invasive measurement of arterial pressure wave reflections and pulse wave velocity. Artery Research 2009;3(2):79-88.

6. Mohiaddin RH, Firmin DN, Longmore DB. Age-related changes of human aortic flow wave velocity measured noninvasively by magnetic resonance imaging. Journal of Applied Physiology 1993;74(1):492-497.

7. Dumoulin C, Doorly D, Caro C. Quantitative measurement of velocity at multiple positions using comb excitation and Fourier velocity encoding. Magnetic resonance in medicine 1993;29(1):44-52.

8. Hardy CJ, Bolster BD, McVeigh ER, Adams WJ, Zerhouni EA. A one - dimensional velocity technique for NMR measurement of aortic distensibility. Magnetic resonance in medicine 1994;31(5):513-520.

9. Boese J, Bock M, Schoenberg S, Schad L. Estimation of aortic compliance using magnetic resonance pulse wave velocity measurement. Physics in medicine and biology 2000;45(6):1703.

10. Rogers WJ, Hu Y-L, Coast D, Vido DA, Kramer CM, Pyeritz RE, Reichek N. Ageassociated changes in regional aortic pulse wave velocity. Journal of the American College of Cardiology 2001;38(4):1123-1129.

11. Metafratzi ZM, Efremidis SC, Skopelitou AS, De Roos A. The clinical significance of aortic compliance and its assessment with magnetic resonance imaging. Journal of Cardiovascular Magnetic Resonance 2002;4(4):481-491.

12. Fielden SW, Fornwalt BK, Jerosch - Herold M, Eisner RL, Stillman AE, Oshinski JN. A new method for the determination of aortic pulse wave velocity using cross correlation on 2D PCMR velocity data. Journal of Magnetic Resonance Imaging 2008;27(6):1382-1387.

13. Grotenhuis HB, Westenberg JJ, Steendijk P, van der Geest RJ, Ottenkamp J, Bax JJ, Jukema JW, de Roos A. Validation and reproducibility of aortic pulse wave velocity as assessed with velocity - encoded MRI. J Magn Reson Imaging 2009;30(3):521-526. 
14. Markl M, Wallis W, Brendecke S, Simon J, Frydrychowicz A, Harloff A. Estimation of global aortic pulse wave velocity by flow-sensitive 4D MRI. Magn Reson Med 2010;63(6):1575-82.

15. Taviani V, Patterson AJ, Graves MJ, Hardy CJ, Worters P, Sutcliffe MP, Gillard JH. Accuracy and repeatability of fourier velocity encoded $\mathrm{M}$ - mode and two dimensional cine phase contrast for pulse wave velocity measurement in the descending aorta. Journal of Magnetic Resonance Imaging 2010;31(5):11851194.

16. Taviani V, Hickson SS, Hardy CJ, McEniery CM, Patterson AJ, Gillard JH, Wilkinson IB, Graves MJ. Age - related changes of regional pulse wave velocity in the descending aorta using Fourier velocity encoded M - mode. Magnetic Resonance in Medicine 2011;65(1):261-268.

17. Markl M, Wallis W, Strecker C, Gladstone BP, Vach W, Harloff A. Analysis of pulse wave velocity in the thoracic aorta by flow - sensitive four - dimensional MRI: Reproducibility and correlation with characteristics in patients with aortic atherosclerosis. Journal of Magnetic Resonance Imaging 2012;35(5):1162-1168.

18. Wentland AL, Wieben O, Francois CJ, Boncyk C, Munoz Del Rio A, Johnson KM, Grist TM, Frydrychowicz A. Aortic pulse wave velocity measurements with undersampled 4D flow-sensitive MRI: comparison with 2D and algorithm determination. J Magn Reson Imaging 2012;37(4):853-859.

19. Roccabianca S, Figueroa C, Tellides G, Humphrey J. Quantification of regional differences in aortic stiffness in the aging human. Journal of the mechanical behavior of biomedical materials 2014;29:618-634.

20. Latham RD, Westerhof N, Sipkema P, Rubal BJ, Reuderink P, Murgo JP. Regional wave travel and reflections along the human aorta: a study with six simultaneous micromanometric pressures. Circulation 1985;72(6):1257-1269.

21. Mitchell GF, Pfeffer MA, Finn PV, Pfeffer JM. Comparison of techniques for measuring pulse-wave velocity in the rat. Journal of Applied Physiology 1997;82(1):203-210.

22. Millasseau SC, Stewart AD, Patel SJ, Redwood SR, Chowienczyk PJ. Evaluation of Carotid-Femoral Pulse Wave Velocity: Influence of Timing Algorithm and Heart Rate. Hypertension 2005;45(2):222-226.

23. Dyverfeldt P, Kvitting JPE, Sigfridsson A, Engvall J, Bolger AF, Ebbers T. Assessment of Fluctuating Velocities in Disturbed Cardiovascular Blood Flow: InVivo Feasibility of Generalized Phase-Contrast MRI. J Magn Reson Imaging 2008;28(3):655-663.

24. Wigström L, Ebbers T, Fyrenius A, Karlsson M, Engvall J, Wranne B, Bolger AF. Particle trace visualization of intracardiac flow using time-resolved 3D phase contrast MRI. Magnetic Resonance in Medicine 1999;41(4):793-799.

25. Ebbers T, Haraldsson H, Dyverfeldt P, Sigfridsson A, Warntjes M, Wigström L. Higher order weighted least-squares phase offset correction for improved accuracy in phase-contrast MRI. 2007; Berlin, Germany. p 1367.

26. Westenberg JJ, de Roos A, Grotenhuis HB, Steendijk P, Hendriksen D, van den Boogaard PJ, van der Geest RJ, Bax JJ, Jukema JW, Reiber JH. Improved aortic pulse wave velocity assessment from multislice two - directional in - plane velocity - encoded magnetic resonance imaging. Journal of Magnetic Resonance Imaging 2010;32(5):1086-1094.

27. Kroon DJ. 2009 Nov 9, 2012. Accurate Fast Marching. MATLAB Central File Exchange. Nov 9, 2012. 
28. Nichols W, McDonald D. Wave-velocity in the proximal aorta. Medical and biological engineering 1972;10(3):327-335.

29. Nichols W, O'Rourke M, Vlachopoulos C. McDonald's Blood Flow in Arteries. London: Hodder Arnold; 2011.

30. Asmar R, Benetos A, Topouchian J, Laurent P, Pannier B, Brisac A-M, Target R, Levy BI. Assessment of arterial distensibility by automatic pulse wave velocity measurement validation and clinical application studies. Hypertension 1995;26(3):485-490.

31. Latson TW, Hunter WC, Katoh N, Sagawa K. Effect of nitroglycerin on aortic impedance, diameter, and pulse-wave velocity. Circulation research 1988;62(5):884-890.

32. Bland J, Altman D. Statistical methods for assessing agreement between two methods of clinical measurement. Lancet 1986;1(8476):307-310.

33. Åstrand H, Stålhand J, Karlsson J, Karlsson M, Sonesson B, Länne T. In vivo estimation of the contribution of elastin and collagen to the mechanical properties in the human abdominal aorta: effect of age and sex. Journal of applied physiology 2011;110(1):176-187. 
TABLES

Table 1. Demographics

\begin{tabular}{|c|c|c|c|c|c|}
\hline & Age (years) & Height $(\mathrm{cm})$ & Weight (kg) & $\begin{array}{l}\text { Blood pressure } \\
(\mathrm{mmHg})^{*}\end{array}$ & $\begin{array}{l}\text { Heart rate } \\
(\mathrm{bpm})^{* *}\end{array}$ \\
\hline \multirow{8}{*}{ 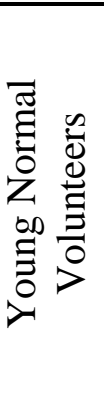 } & 23 & 181 & 69 & $118 / 70$ & 64 \\
\hline & 24 & 185 & 77 & $131 / 67$ & 59 \\
\hline & 21 & 182 & 67 & $134 / 74$ & 68 \\
\hline & 23 & 186 & 73 & $125 / 58$ & 61 \\
\hline & 24 & 187 & 72 & $116 / 73$ & 63 \\
\hline & 26 & 178 & 71 & $115 / 65$ & 55 \\
\hline & 21 & 188 & 72 & $125 / 65$ & 65 \\
\hline & 24 & 179 & 75 & $133 / 73$ & 65 \\
\hline \multirow{8}{*}{ 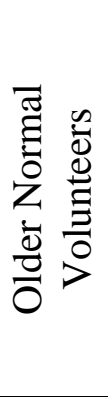 } & 56 & 187 & 90 & $121 / 78$ & 50 \\
\hline & 56 & 182 & 72 & $132 / 78$ & 67 \\
\hline & 55 & 180 & 87 & $133 / 82$ & 64 \\
\hline & 57 & 190 & 78 & $143 / 88$ & 48 \\
\hline & 60 & 167 & 61 & $128 / 76$ & 60 \\
\hline & 58 & 178 & 72 & $123 / 74$ & 68 \\
\hline & 58 & 182 & 85 & $127 / 78$ & 60 \\
\hline & 60 & 173 & 81 & $131 / 74$ & 54 \\
\hline
\end{tabular}

* Systolic/diastolic blood pressure measured over the left arm prior to the MRI exam. ** Heart rate during MRI

Table 2. PWV for the different age groups, locations and methods evaluated in this study.

\begin{tabular}{lcccccc}
\hline & FA & XC & COM & TTU & TTF & TTF2 \\
\hline PWV_global_young & $5.7 \pm 0.7$ & $5.5 \pm 0.7$ & $6.0 \pm 0.7$ & $3.9 \pm 0.4$ & $3.6 \pm 0.3$ & $3.7 \pm 0.3$ \\
PWV_global_old & $9.3 \pm 1.3$ & $8.9 \pm 1.4$ & $9.2 \pm 1.3$ & $6.3 \pm 2.3$ & $6.4 \pm 1.7$ & $6.8 \pm 2.6$ \\
PWV_prox_young & $4.3 \pm 0.8$ & $4.2 \pm 0.7$ & $4.6 \pm 0.9$ & $3.3 \pm 0.6$ & $3.7 \pm 0.4$ & $4.2 \pm 0.7$ \\
PWV_prox_old & $8.8 \pm 1.6$ & $10.2 \pm 3.6$ & $9.1 \pm 2.1$ & $5.7 \pm 2.2$ & $8.8 \pm 3.5$ & $8.9 \pm 4.5$ \\
PWV_mid_young & $6.6 \pm 2.2$ & $5.7 \pm 1.6$ & $6.3 \pm 1.8$ & $4.0 \pm 0.7$ & $3.3 \pm 0.5$ & $3.3 \pm 0.6$ \\
PWV_mid_old & $11.2 \pm 3.7$ & $10.6 \pm 3.8$ & $10.5 \pm 3.1$ & $7.2 \pm 4.5$ & $7.0 \pm 2.1$ & $7.7 \pm 4.2$ \\
PWV_dist_young & $5.1 \pm 0.7$ & $4.6 \pm 0.8$ & $5.8 \pm 1.0$ & $5.6 \pm 1.6$ & $4.5 \pm 0.8$ & $4.9 \pm 1.3$ \\
PWV_dist_old & $7.3 \pm 1.7$ & $6.9 \pm 1.7$ & $7.6 \pm 1.7$ & $5.5 \pm 1.7$ & $5.2 \pm 1.5$ & $5.6 \pm 2.5$ \\
\hline
\end{tabular}

Table 3. Age-related descending aortic PWV in the present study compared to previous studies with higher temporal resolution methods ${ }^{13,16}$.

\begin{tabular}{|c|c|c|c|c|}
\hline Study & Method & $\begin{array}{c}\text { Temporal } \\
\text { resolution } \\
{[\mathrm{ms}]}\end{array}$ & $\begin{array}{c}\text { Age } \\
(\text { mean } \pm 1 \mathrm{SD}) \\
\text { [years] }\end{array}$ & $\begin{array}{l}\text { Global PWV in desc ao } \\
(\text { mean } \pm 1 \mathrm{SD})[\mathbf{m} / \mathbf{s}]\end{array}$ \\
\hline Present study & 4D flow MRI & $39-43$ & $23 \pm 2$ & $3.6 \pm 0.4$ \\
\hline Grotenhuis et al. ${ }^{13}$ & 2D through-plane PC-MRI & $6-10$ & $29 \pm 8$ & $4.3 \pm 0.6$ \\
\hline Taviani et al. ${ }^{16}$ & 1D FVE MRI & 3.5 & $\sim 30^{*}$ & $4.2 \pm 0.7$ \\
\hline Taviani et al. ${ }^{16}$ & 1D FVE MRI & 3.5 & $\sim 50^{*}$ & $5.9 \pm 0.9$ \\
\hline Present study & 4D flow MRI & $39-43$ & $58 \pm 2$ & $6.4 \pm 1.7$ \\
\hline Taviani et al. 16 & 1D FVE MRI & 3.5 & $\sim 70^{*}$ & $8.0 \pm 1.3$ \\
\hline
\end{tabular}

* Approximate mean age calculated as the average of the age ranges reported by Taviani et al ${ }^{16}$. 


\section{FIGURE LEGENDS}

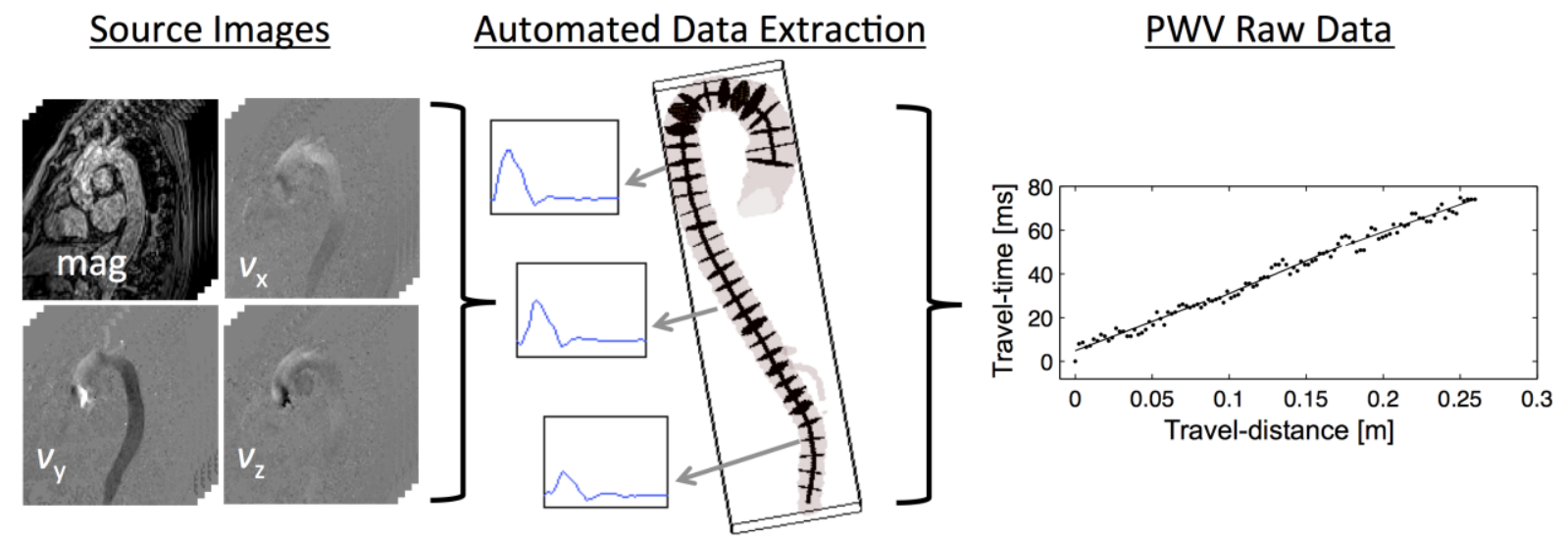

Figure 1. Schematic of semiautomatic 4D flow MRI-based estimation of regional PWV based on extraction of flow waveforms throughout a vessel of interest. Velocity and magnitude images (left) are combined to generate a PC-MR angiogram that is used for segmentation of the aortic lumen. A centerline is extracted from the segmentation and this centerline is used to define locations for extraction of pulsatile flow waveforms throughout the aorta (middle). The travel-time and travel-distance variables are calculated and subsequently combined in a 2D graph that can be interrogated for regional PWV estimation (right).
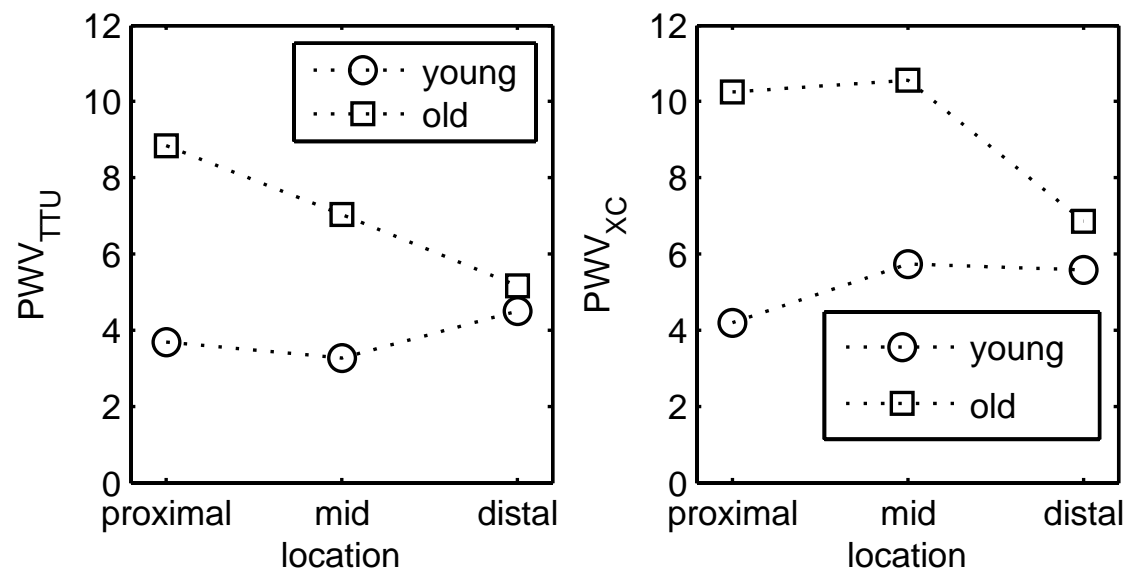

Figure 2. Profile plots of group means of regional PWV as measured with the TTF (left) and $\mathrm{XC}$ (right) methods in young (circles) and older (squares) subjects. For both methods, the 
location-dependency of PWV is more pronounced in the older compared to younger volunteers, the age-dependency is more pronounced in the proximal compared to distal suprarenal descending aorta, and there is an interaction between age and location.

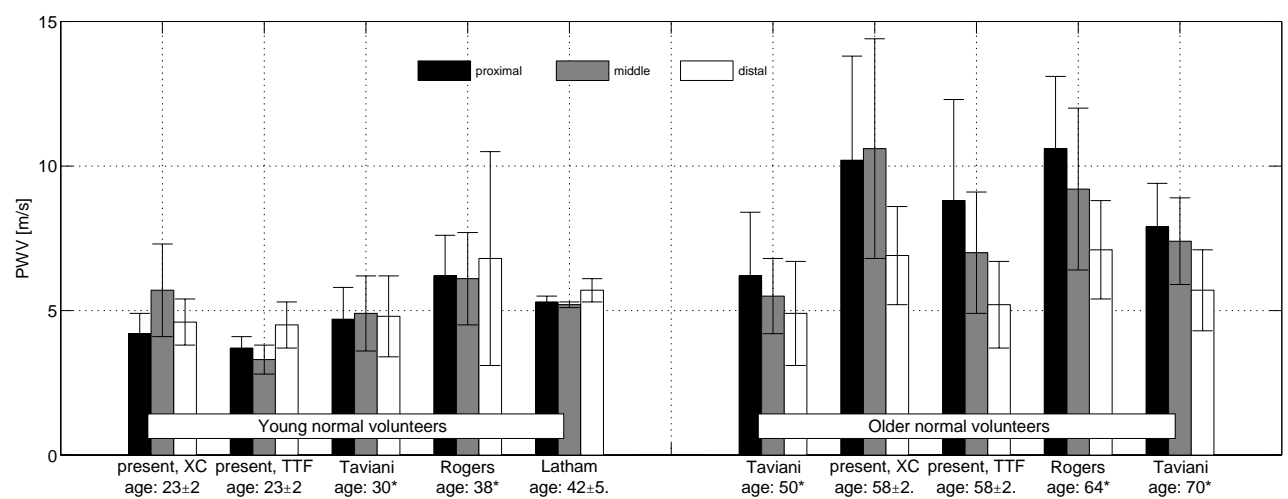

Figure 3. Age-related regional PWV in the present study as obtained with the TTF and XC methods are compared against results previously published by Latham et al ${ }^{20}$, Rogers et al ${ }^{10}$, and Taviani et al ${ }^{16}$. Left: PWV data for the proximal (black), mid (gray) and distal (white) descending aorta in younger subjects ( $<50$ y.o.). Right: corresponding data in older subjects (>50 y.o.). The mean age (*approximated mean age) is indicated below each triplet of bars. 